\section{Endoparasites in wild animals at the zoological garden in Skopje, Macedonia}

\section{Elena Atanaskova ${ }^{1}$, Zoran Kochevski ${ }^{2}$, Jovana Stefanovska ${ }^{3} \&$ Goran Nikolovski ${ }^{4}$}

1,4 Department of Health Preventive for Pets and Ungulates, ${ }^{2,3}$ Department of Parasitology and Parasitic Diseases, Faculty of Veterinary Medicine, LazarPop-Trajkov 5-7, 1000 Skopje, Republic of Macedonia Email: ${ }^{1}$ eatanaskova@fvm.ukim.edu.mk (corresponding author), ${ }^{2}$ zkochevski@fvm.ukim.edu.mk, ${ }^{3}$ jstefanovska@fvm. ukim.edu.mk, ${ }^{4}$ gnikolovski@fvm.ukim.edu.mk

Parasitic diseases play an important role for wild animals in captivity. In captivity the health status of the animals depends on many factors, like feeding, keeping conditions, animal management and environmental conditions such as temperature and humidity. The staff plays an important role in the transmission of parasites amongst animals in a zoo, through their shoes, clothes, hands, food or with working tools. Another possibility of parasite transmission is the animals themselves, when they are moved from one enclosure to another, without proper parasite treatment. Mixing different species brings additional risks of parasitic infections. In the wild, animals might have a natural resistance against parasitic infections or live in a balanced system with their parasites. But the change in environment and

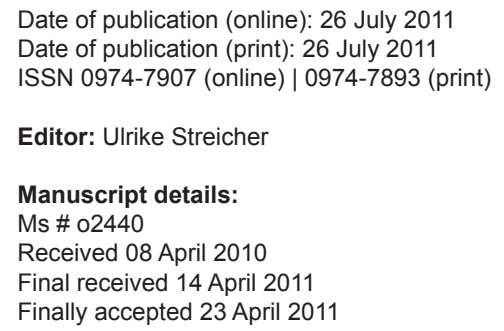

Citation: Atanaskova, E., Z. Kochevski, J. Stefanovska \& G. Nikolovski (2011). Endoparasites in wild animals at the zoological garden in Skopje, Macedonia. Journal of Threatened Taxa 3(7): 1955-1958.

Copyright: (c) Elena Atanaskova, Zoran Kochevski, Jovana Stefanovska \& Goran Nikolovski 2011. Creative Commons Attribution 3.0 Unported License. JoTT allows unrestricted use of this article in any medium for non-profit purposes, reproduction and distribution by providing adequate credit to the authors and the source of publication.

Acknowledgements: We would like to thank Vesna Levajkovic, DVM from the zoological garden in Skopje, Macedonia for helping in the sample collection.

OPEN ACCESS I FREE DOWNLOAD living conditions from freedom to captivity influences the animals' ecology and might increase the

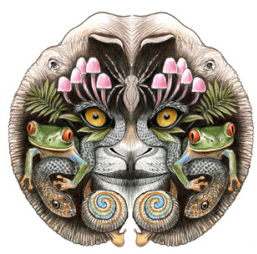
sensitivity for parasitic infections (Goossensa et al. 2005). Parasitic diseases are one of the main causes of death in wild animals in captivity (Rao \& Acharjyo 1984). In addition, some parasites are zoonotic and are a risk to human health (Maske et al. 1990; Chakraborty et al. 1994; Kashid et al. 2003).

For these reasons we consider it very important to conduct preventive measures, to regularly control the presence of parasites in the animals and to undertake adequate therapy when required. Skopje Zoological Garden, Macedonia implements a regular deworming program at least once a year. For several years they have used different antiparasitic drugs for different groups of animals such as ivermectin, piperazine citrate, fenbendazol (Panacur), praziquantel, and pyrantel (Biheldon).

The goal of our study was to evaluate the presence of gastrointestinal parasites in the animals in the Zoological Garden in Skopje, Macedonia.

Materials and methods: The study was conducted at the Zoological Garden in Skopje, established in 1926 on an area of 12 acres with a collection of 300 animals from 56 different species. On several occasions animals were treated in November and then samples were taken in the following April.

Fecal samples were taken over a period of three years from 28 different species of animals (Table 1). The samples were always collected in April. The samples were brought to the laboratory for parasitology and parasitic diseases at the faculty of veterinary medicine in Skopje, in portable refrigerators. Fecal examination was performed by flotation method using $\mathrm{ZnSO}_{4}$ with a specific gravity of $1.18-1.20$ (371g zinc sulfate in $1000 \mathrm{ml}$ water). From every animal $2-5 \mathrm{~g}$ of feces were mixed with $10 \mathrm{ml} \mathrm{ZnSO}_{4}$ then the sample was centrifuged at $1200 \mathrm{rpm}$ for 5 minutes. Every sample was checked under the microscope at $40 \mathrm{X}$ enlargement (Dryden et al. 2005).

We divided the examined animals into three groups according to the type of enclosure they were kept in. These groups did not consider the animals' age and there was no control group.

The first group consisted of animals that were kept in indoor enclosures - such as the menagerie for 
Table 1. Results of examination of the first, second and third group.

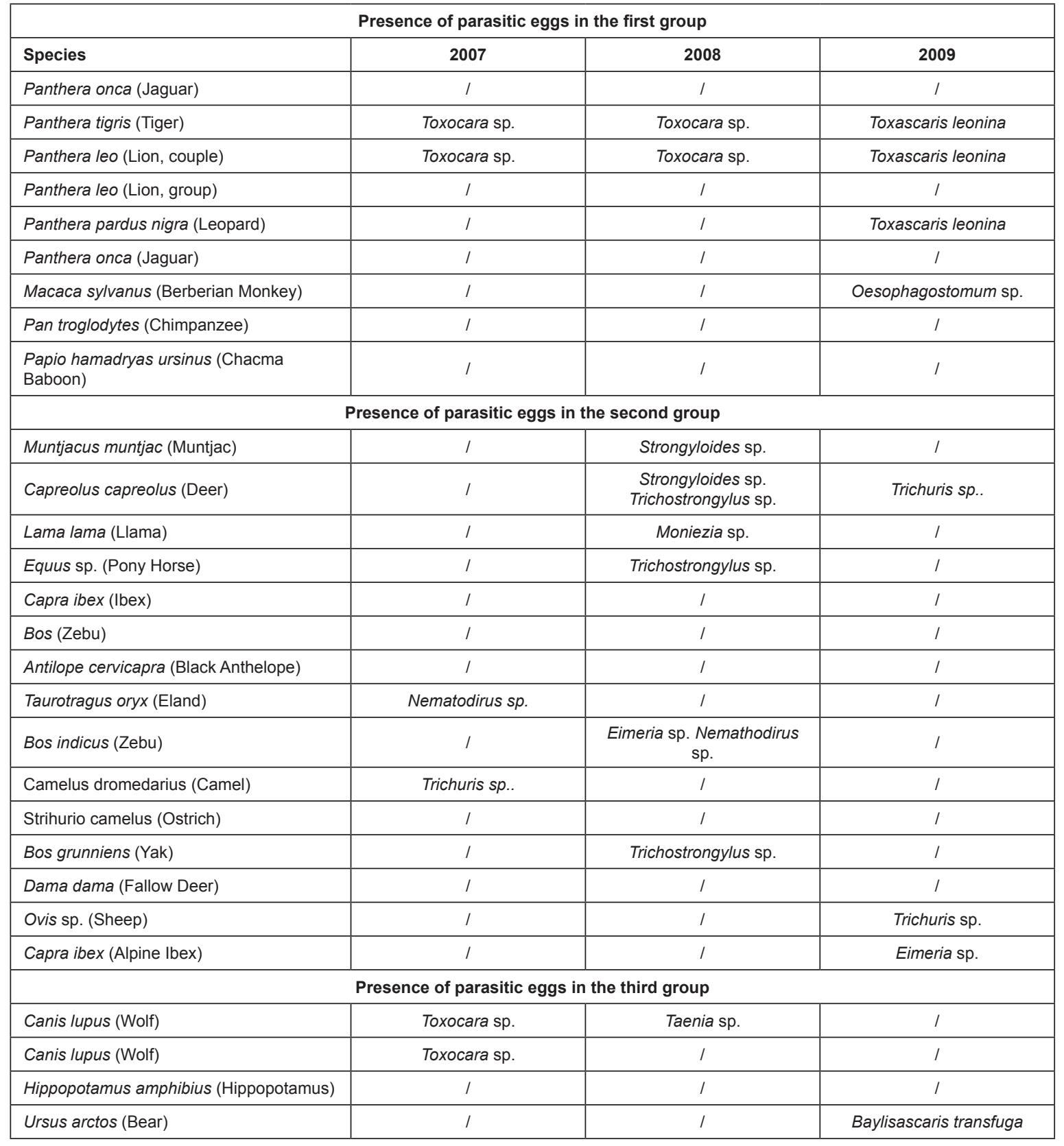

wild cats and the ape enclosure. The second group comprised animals like camel, ostrich and ibex that were held in outdoor enclosures with open soil. The third group included animals held in semi-open enclosures: bears, wolves and hippopotamus. In the semi-open enclosures animals were closed in cages during the cold season in winter and were free to go outside in the other periods of the year.

In the first group parasite treatment was performed in May using piperazine citrate $(2.5 \mathrm{mg} / \mathrm{kg})$ (Jacobs 1987) in all investigated years.
Animals in the second group were treated with ivermectin $(0.2 \mathrm{mg} / \mathrm{kg}$ ) (Bowman 1995), twice in 2007 (May and November), and once in 2008 (May). In 2009 animals were treated twice with piperazine citrate $(110 \mathrm{mg} / \mathrm{kg}$ ) at an intervall of four weeks (May and June) (Gibson 1957).

The third group was treated once in May in 2007 with ivermectin $(0.2 \mathrm{mg} / \mathrm{kg}$ ) (Bowman 1995). In 2008 the treatment was applied again twice, in May and November, also using ivermectin. In 2009, two treatments were applied, first a praziquantel $(5 \mathrm{mg} / \mathrm{kg})$ 
and pyrantel $(5 \mathrm{mg} / \mathrm{kg}$ ) combination was used (Bowman 1995) in May, and after six months fenbendazol $(50 \mathrm{mg} /$ $\mathrm{kg}$ ) was applied (Bowman 1992).

Results: Eggs of the following parasites were identified: Baylisascaris transfuga, Eimeria sp., Moniezia sp., Nemathodirus sp., Oesophagostomum sp., Strongyloides sp., Taenia sp., Toxocara sp., Toxascaris leonina, Trichuris sp., Trichostrongylus sp.

Within the first group we found parasite eggs in Panthera tigris and Panthera leo in all three years consecutively. Panthera pardus nigra and Macaca sylvanus were parasite free in 2007 and 2008 but showed parasitic infection in 2009. In the second group most of the animals were found parasite positive in 2008, but in 2009 most of the animals were free of parasites. In the third group, Canis lupus was found positive for Toxocara spp. In the following years the animals were found free of this parasite. The infection of Ursus arctos with Baylisascaris transfuga which was found in 2009 was probably a result of the introduction of a new bear from the wild and insufficient cleaning measures.

Discussion: Helminthoses are a big problem in zoo animals. In captivity animals appear to be less resistant to parasitic infections than in their natural habitats. Our study shows that the number of infected animals in the whole zoological garden in Skopje is fairly high with an infection rate of $21.4 \%, 32.1 \%$ and $28.6 \%$ in the years 2007, 2008 and 2009. A comparable study by Lalošević et al. (2007) found an infection rate of $17.2 \%$ in 75 samples of animals kept at Palic Zoo in Serbia, which is considerably lower.

Some parasites (geohelminths) potentially accumulate in a captive environment, in particular in open soil enclosures, which cannot be easily disinfected. Their survival in the soil is strongly impacted by climatic factors. Other parasites require an intermediate host and are less likely to accumulate in a captive environment, because their intermediate host might not occur in the enclosure (Lalošević et al. 2007). Our results confirmed this finding: all parasites found during the examinations are geohelminths, which do not require an intermediate host. This has a very important epidemiological meaning and our results are similar with the results of other studies. In 2007 and 2008 the percentage of infected animals was identical in animals kept in indoor enclosures all year round (group 1), while in 2009 it was double despite the parasite treatment. Though it is possible that the animals were parasite free immediately after the treatment there is obviously a high rate of reinfection (Table 2).

Animals living in outdoor open soil enclosures (group 2) were treated twice in 2007 while in 2008 only once. However the infection rate increased from 2007 to 2008, while it decreased from 2008 to 2009. The change in infection rate cannot be explained by the parasite treatment. We do not know if the animals were parasite free immediately after our treatment. Whatever effect there might have been the reinfection rate under this keeping conditions is very high.

Within this group we found eggs of Toxocara spp. and Toxascaris leonina in tigers. The tigers were treated both in 2007 and 2008 with piperazine citrate, but the same parasites were still found in 2009. Toxocara and Toxascaris have very high tenacity and their presence during the 3-year research is a sign that the preventive measures applied during this period are not sufficient or that there is a high rate of reinfection. In 2009 the tigers were treated for three consequent days with fenbendazol $(10 \mathrm{mg} / \mathrm{kg})$ hoping that this will be a more efficient medication. Animals living part time in indoor enclosures and part time in outdoor enclosures (group 3) received two treatments every year during our study and showed the lowest rate of infection. However looking at results from group 1, two treatments alone are not necessarily sufficient to reduce the parasites. It is likely that the management of shifting enclosures every few months contributes to the reduction of parasites.

It is difficult to draw detailed conclusions from our study for various reasons. Firstly the time between the deworming and the fecal sampling is very long and ranges between 6 and 11 months. Even if the treatment was initially effective, in such a long time there is a high risk of reinfection via above mentioned vectors as personal or tools. In addition due to the reconstruction

Table 2. Percent of infected animals by groups.

\begin{tabular}{|c|c|c|c|}
\hline Group & $\mathbf{2 0 0 7}$ & $\mathbf{2 0 0 8}$ & $\mathbf{2 0 0 9}$ \\
\hline 1 & $22.2 \%$ & $22.2 \%$ & $44.4 \%$ \\
\hline 2 & $13.3 \%$ & $40 \%$ & $20 \%$ \\
\hline 3 & $40 \%$ & $25 \%$ & $25 \%$ \\
\hline
\end{tabular}


of the enclosures during the past three years, many animals were transferred from one enclosure to another and were mixed with other species of animals. This might be the reason why despite antiparasitic treatment in some species of animals, different species of parasites were found each year.

To control parasitic infections it is necessary to undertake appropriate antiparasitic therapy, to increase cage hygiene and to introduce good animal and staff management. It should also be kept in mind that every antiparasitic therapy might potentially cause additional stress in the animal and increase the possibility of infection. Regular parasite controls of food and water should also be conducted; quality food and appropriate addition of vitamins and minerals is an additional measure to reduce the risk of parasitic infections (Borghare et al. 2009).

\section{REFERENCES}

Borghare, A.T., V.P. Bagde, A.D. Jaulkar, D.D. Katre, P.D. Jumde, D.K. Maske \& G.N. Bhangale (2009). Incidence of gasrointestinal helminthiasis in captive deers at Nagpur. Veterinary World 2(9): 337-338.

Bowman, D.D. (1992). Anthelmintics for dogs and cats effective against nematodes and cestodes. The compendium on continuing education for the practicing veterinarian 14(5): 597-601.

Bowman, D.D. (1995). Georgis' Parasitology for Veterinarians -6th Edition. W.B. Saunders Company, USA, 265pp.
Chakraborty, A., A.R. Gogoi \& B. Choudhary (1994). Prevalence of parasitic infection in captive wild herbivores in a zoo in Assam, India. Indian Journal of Animal Science 9: $149-152$.

Dryden, M.W., A. Payne \& S. Ridley (2005). Comparison of common fecal flotation techniques for the recovery of parasite eggs and oocysts. Veterinary Therapeutics 6(1): 14-28.

Gibson, T.E. (1957). Critical test of piperazine adipate as an equine anthelmintic. British Veterinary Journal 113: 99092.

Goossensa, E., P. Dornya, J. Boomkerd, F. Vercammen \& Vercruysse (2005). A 12-month survey of the gastrointestinal helminths of antelopes, gazelles and giraffes kept at two zoos in Belgium. Veterinary Parasitology 127: 303-312.

Jacobs, D.E. (1987). Antehlminitcs for dogs and cats. International Journal for Parasitology17(2): 511-518.

Kashid, K.P., G.B. Shrikhande \& G.R. Bojne (2003). Incidence of gastro-intestinal helminths captive wild animals at different locations. Zoos' Print Journal 18(3): 1053-1054.

Lalošević, V., D. Lalošević, S. Boboš, M. Šinković \& L. Spasojević (2007). Nalaz crevnih parazita kod životinja u zoološkom vrtu 'Palić', Savremena poljoprivreda 56 (3-4): 98-102.

Maske, D.K., N.C. Bhilegaonkar \& M.R. Sardey (1990). Helminth parasites in zoo animals of Maharajbagh, Nagpur, Maharashtra State. Indian Journal of Animal Science 5: 277-278.

Rao,A.T. \& L.N. Acharjyo (1984). Diagnosis and classification of common diseases of captive animals at Nandankanan Zoo in Orissa (India). Indian Journal of Animal Health 33: 147-152. 